\title{
论 文
}

\section{非离子型表面活性剂Tween 60用于碳纳米管的双 水相分离}

陈少闯 ${ }^{1}$, 吕敏 ${ }^{1,2 *}$, 李彦 ${ }^{1 *}$

1. 北京分子科学国家研究中心, 纳米器件物理与化学教育部重点实验室, 稀土材料化学及应用国家重点实验室, 北京大学化学与分子工程学 院, 北京 100871

2. 北京大学深圳研究院, 深港产学研基地, 深圳 518057

*通讯作者, E-mail: yanli@pku.edu.cn; 1vmin@pku.edu.cn

收稿日期: 2021-08-20; 接受日期: 2021-09-26; 网络版发表日期: 2021-11-23

科技部重点研发计划课题(编号: 2016YFA0201904)、国家自然科学基金委员会项目(编号: 22120102004, 21631002)、深圳市科技计划项目(编 号: JCYJ20170817113121505)、深圳市海外高层次人才创新创业专项资金(编号: KQTD20180411143400981)和北京分子科学国家研究中心(编 号: BNLMS- CXTD-202001)资助项目

摘要单壁碳纳米管以其优异的电学和光学性能受到了广泛的关注，高性能器件等应用要求使用性质均一的 单壁碳纳米管。因此，不同结构的单壁碳纳米管的分离具有重要意义. 双水相萃取是一种能够对单壁碳纳米管进 行结构分离的新方法, 分离结果稳定可靠, 且不需要复杂的设备, 具有简捷、高效、易扩大规模等特点. 本文通过 调节脱氧胆酸钠(DOC) 和非离子型表面活性剂Tween 60 的浓度, 实现了单壁碳纳米管的管径分离及高纯度 $(6,5)$ 的 分离. 分离可以在较高的表面活性剂浓度下进行, 提高DOC与Tween 60 对单壁碳纳米管表面的竞争包覆, 进一步 提高复合表面活性剂体系分离效果. Tween 60-DOC复合表面活性剂体系与SDS-DOC复合表面活性剂体系中的分 离结果对比表明, Tween 60 非离子表面活性剂的引入有利于分离得到更高纯度的 $(6,5)$ 单壁碳纳米管.

关键词单壁碳纳米管, 双水相萃取, 非离子表面活性剂

\section{1 引言}

单壁碳纳米管自 1993 年 ${ }^{[1]}$ 被发现以来, 以其优异的 电学、光学等性质受到了广泛的关注, 在高性能电子 器件 ${ }^{[2,3]}$ 、高分辨生物成像 ${ }^{[4]}$ 、单光子发射 ${ }^{[5]}$ 等领域有 着巨大的应用潜能. 然而, 单壁碳纳米管的性质依其结 构而改变，在应用中结构和性质均一的单壁碳纳米管 更有利于发挥其优异的性能，因此可控地制备及分离 特定结构的单壁碳纳米管是当下亟需解决的重要问题.
在单壁碳纳米管被发现的 10 年后才陆续有关于单 壁碳纳米管分离的相关报道. 目前, 对水溶液中碳纳米 管进行分离的方法主要有离子交换色谱法 ${ }^{[6 ~ 8]}$ 、密度 梯度离心法 ${ }^{[9 \sim 11]}$ 、凝胶色谱法 ${ }^{[12 \sim 14]}$ 以及双水相萃取 法 $^{[15 ~ 19]}$. 最初都只能做到管径分离或金属性/半导体性 分离, 随后通过优化条件能够部分实现手性分离.

2013年, 双水相体系首次被Khripin等 ${ }^{[15]}$ 应用于单 壁碳纳米管的分离. 他们发现将胆酸钠 $(\mathrm{SC})$ 分散的单 壁碳纳米管与聚乙二醇/葡聚糖(PEG/DX)双水相体系

引用格式: Chen S, Lyu M, Li Y. Aqueous two-phase extraction of single-walled carbon nanotubes using nonionic surfactant Tween 60. Sci Sin Chim, 2022, 52: 102-107, doi: 10.1360/SSC-2021-0184 
混合, 之后再加入十二烷基硫酸钠(SDS), 单壁碳纳米 管会在上相和下相间产生自发的分配. 此后, Fagan 等 ${ }^{[18]}$ 通过调控体系中 SDS 与脱氧胆酸钠(DOC) 的浓度, 实现了依据管径大小对单壁碳纳米管进行分离, 通过 多步分离得到了几种高手性纯度的小管径碳纳米管. 随后, 基于此方法, 他们又实现了大管径碳纳米管的分

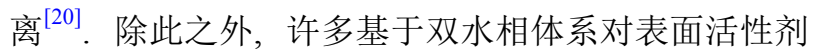
分散的碳纳米管的分离工作也实现了单壁碳纳米管的 导电性分离 ${ }^{[21]}$ 和部分单手性单壁碳纳米管的分 离 $^{[22,23]}$. 由于此方法适于连续多步操作, 它与逆流色谱 技术的联用实现了碳纳米管的连续自动多步分离 ${ }^{[24]}$. 双水相萃取分离结果稳定可靠, 不需要复杂的设备, 具 有简捷、高效、易扩大规模等特点.

目前已经可以用表面活性剂 ${ }^{[15,18,19,21 ~ 23,25]}$ 、单链 DNA ${ }^{[16,17,26]}$ 等分散剂在双水相体系中分离出 20 余种 ${ }^{[27]}$ 高纯度单手性碳纳米管. 但是, 特定序列的DNA价格 较昂贵, 因此探索基于表面活性剂的分散剂体系就显 得更具实际应用价值. 目前, 常用的表面活性剂多是 阴离子型表面活性剂, 如DOC、SC、SDS、十二烷基 苯磺酸钠(SDBS)等, 而非离子型表面活性剂因为在早 年用于分散单壁碳纳米管的效果不如 DOC 和 SC 等 ${ }^{[28,29]}$, 近年来末见用于双水相分离的报道. 但非离子 表面活性剂受溶液环境影响小, 而且各种表面活性剂 具有不同的性质，对分离也会有不同的影响，可以获 得不同的分离选择性, 因此研究非离子表面活性剂在 碳纳米管分离中的应用仍有重要意义.

本文将非离子型表面活性剂Tween 60与常用表面 活性剂DOC复配, 用于单壁碳纳米管的双水相萃取分 离, 通过调控表面活性剂的浓度, 实现了管径分离和 手性分离. 与SDS-DOC体系相比, Tween 60 可在高 DOC浓度下分离单壁碳纳米管, 提高DOC与Tween 60 对单壁碳纳米管表面的竞争包覆, 进一步提高分离 效果.

\section{2 实验部分}

\section{1 实验试剂}

CoMoCAT-SG65i单壁碳纳米管(简称SG65i, $\geq 95 \%$ carbon basis ( $\geq 95 \%$ as carbon nanotubes), $0.78 \mathrm{~nm}$ average diameter, Sigma-Aldrich, 美国); 十二 烷基硫酸钠(SDS, $\geq 99 \%$, Sigma-Aldrich); 脱氧胆酸钠
(DOC, BioXtra, $\geq 98.0 \%$, Sigma-Aldrich); 吐温60 (Tween 60, 聚氧乙烯山梨聚糖单硬脂酸酯, TCI chemicals); 聚乙二醇( $\mathrm{PEG}$, 分子量6 kDa, 购自Alfa Aesar); 葡聚糖(DX, 分子量 $70 \mathrm{kDa}$, 购自 $\mathrm{TCI}$ ); 实验所用的超 纯水由 Thermal Scientific ${ }^{\mathrm{TM}}$ Barnstead ${ }^{\mathrm{TM}}$ nanopure超纯 水机制备, 电阻率为 $18.2 \mathrm{M} \Omega \mathrm{cm}$.

\section{2 单壁碳纳米管的分散}

以单壁碳纳米管-DOC 分散液的制备为例。将 $\mathrm{SG} 65 \mathrm{i}$ 单壁碳纳米管直接与水混合, 得到浓度为 $2 \mathrm{mg} / \mathrm{mL}$ 的 SG65i/水悬浊液, 取 $500 \mu \mathrm{L} \mathrm{SG} 65 \mathrm{i} /$ 水悬浊 液, 离心并弃去上层清液. 向沉淀物中加入 $200 \mu \mathrm{L} 5 \%$ $\mathrm{DOC}$ 水溶液和 $800 \mu \mathrm{L}$ 超纯水, 使 $\mathrm{DOC}$ 的质量浓度为 $1 \%$ $(w / w)$, 得到总体积为 $1 \mathrm{~mL}$ 的混合液. 将该混合液在冰 水浴中进行探针超声(Sonics VCX130), 功率为 $9 \mathrm{~W}$, 连 续超声两次, 每次 $45 \mathrm{~min}$. 超声后的溶液平均分为 10 份, 每份约 $100 \mu \mathrm{L}$, 离心 $90 \mathrm{~min}$, 离心力 $17000 \mathrm{G}$ (ThermoFisher Fresco 21离心机). 离心后, 弃去沉淀、取出上层 清液, 得到单壁碳纳米管分散液, 并用于后续分离.

\section{3 双水相溶液的制备及双水相分离步骤}

聚乙二醇、葡聚糖和水以 $8: 14: 78$ (质量比)混合, 得到双水相的浓缩溶液.

将该双水相浓缩溶液与纯水以 $2: 1$ (体积比)混合, 在 $10000 \mathrm{G}$ 下离心 $2 \mathrm{~min}$, 实现双水相体系的快速分相, 得到空白的双水相稀释溶液, 用于后续分离.

混合 $360 \mu \mathrm{L}$ 上述双水相浓缩溶液、 $60 \mu \mathrm{L}$ 单壁碳 纳米管的分散液和 $120 \mu \mathrm{L}$ 水, 得到含有 DOC分散的单 壁碳纳米管的双水相稀释溶液. 同样地, 在 $10000 \mathrm{G}$ 下 离心 $2 \mathrm{~min}$, 实现双水相体系的分相, 取出上相(记为 $n \mathrm{~T}$, $n$ 表示第 $n$ 个分离步骤取出的上相, T表示上相, 下相用 $\mathrm{B}$ 表示), 加入 $270 \mu \mathrm{L}$ 空白上相和含有表面活性剂的水 溶液, 调控体系中复合表面活性剂的浓度, 不断通过 离心分相、取出上相，补充空白上相和含表面活性剂 的水溶液, 进行多步分离, 如图1所示.

\section{4 吸收光谱表征}

吸收光谱测试中一般将分离得到的上相或下相溶 液稀释 10 倍, 注入光程为 $1 \mathrm{~cm}$ 的吸收池中, 通过紫外可 见近红外吸收光谱仪(PerkinElmer Lamda750, 美国)进 行吸收光谱表征, 光谱采集范围通常为200 1400 nm. 


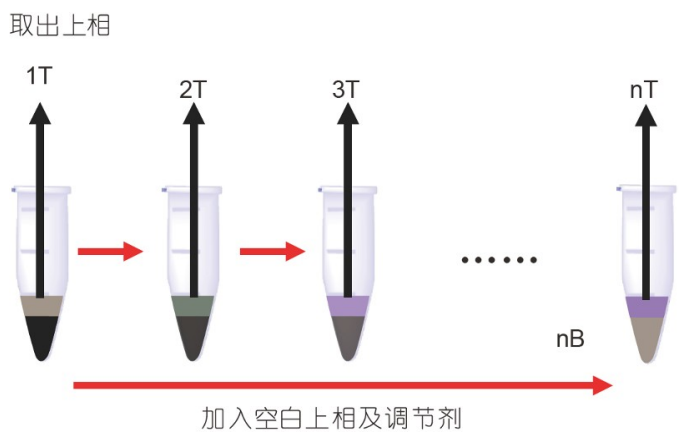

图 1 双水相萃取过程示意图(网络版彩图)

Figure 1 Schematic of aqueous two-phase extraction (color online).

\section{3 结果与讨论}

\subsection{SDS-DOC复合表面活性剂体系}

对于传统的SDS-DOC复合表面活性剂体系，两个 组分DOC和SDS均为离子型表面活性剂, 控制其中的 表面活性剂浓度变化，如图2b所示，取出前几步的上 相，DOC的浓度经过最初的几步降低后基本保持在 $0.02 \%$ 不变，随着SDS浓度的升高，上相单壁碳纳米管 $S_{11}$ 对应的吸收谱峰(800 1300 nm)逐渐向低波长方向 移动(图2a), 说明单壁碳纳米管的管径由大逐渐变小, 有明显的管径分离趋势，并在9T、10T中，即SDS浓度 是DOC浓度的20 30倍时，得到了富集度很高的 $(6,5)$. 这一分离结果与此前文献报道中传统的聚乙二醇-葡 聚糖双水相体系中SDS-DOC的分离趋势 ${ }^{[18]}$ 相符. 公认 的分离机理 ${ }^{[4,14,23]}$ 是DOC更倾向于包覆小管径的单壁 碳纳米管，而SDS更倾向于包覆大管径单壁碳纳米管， 同时SDS包覆的单壁碳纳米管更疏水，在PEG/DX的双 水相体系中更倾向于分配至上相. 因此, 随着SDS浓度 的升高，大管径单壁碳纳米管表面的DOC先是更多地 被SDS取代，从而大管径单壁碳纳米管先分配至上相, 上相中分离得到的单壁碳纳米管管径就逐渐由大变小.

\subsection{Tween 60-DOC复合表面活性剂体系}

将离子型表面活性剂SDS换为Tween 60时, 同样可 以实现单壁碳纳米管的管径分离, 其光学照片如图3e所 示. 与SDS-DOC复合表面活性剂体系的分离策略相同, 降低DOC至较低浓度保持不变，逐步提高Tween 60 的 浓度(图3b)，上相中分离得到的单壁碳纳米管的管径逐 步变小(图3a), 呈现出与SDS-DOC体系相似的管径分离
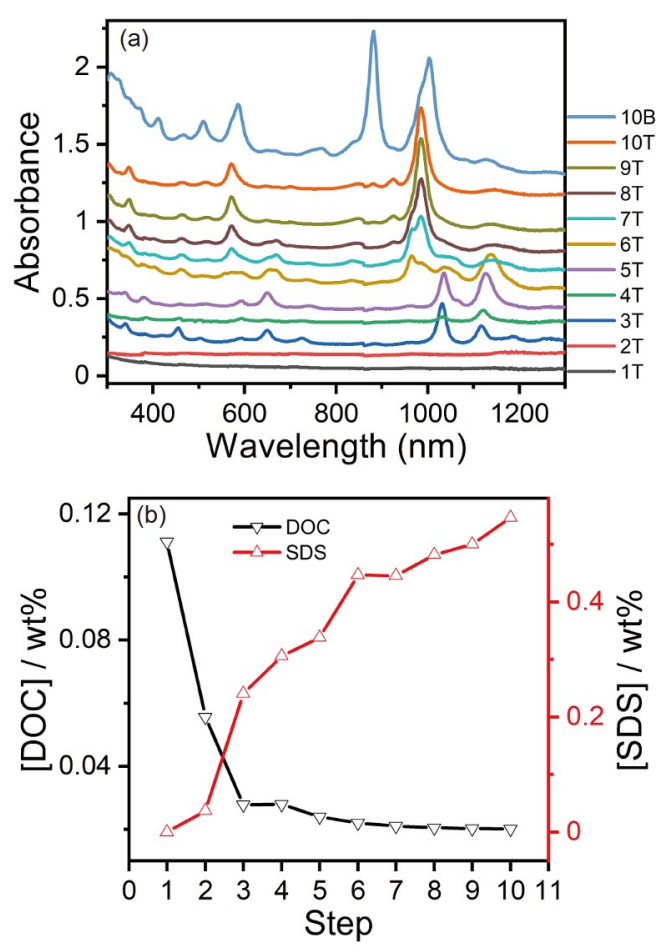

图 2 SDS-DOC复合表面活性剂体系中双水相分离得到各 相的吸收光谱图(a)及分离过程中表面活性剂浓度变化(b) (网络版彩图)

Figure 2 Absorption spectra of each top and bottom phases extracted (a) and mass concentrations of surfactants of the whole ATP system (b) during the ATP extraction in the SDS-DOC co-surfactant system (color online).

的趋势, 说明Tween 60 可能也更倾向于包覆大管径单壁 碳纳米管, 且Tween 60包覆后的单壁碳纳米管更疏水.

当DOC浓度较低 $(0.028 \%)$, Tween 60 与DOC的浓 度比在较低值(1.5)时, 单壁碳纳米管已基本全部分配 至上相, 即可完成分离. 然而, 在SDS-DOC体系中, 保 持DOC浓度在 $0.02 \%$ 左右, SDS浓度需达到DOC浓度 的20 30倍, 才能实现比较完全的分离. 因此, Tween 60 相对 SDS 体系, 有更强的与DOC竞争包覆的能力. 但同时, 多个上相的吸收光谱中出现多种不同手性单 壁碳纳米管的吸收峰, 说明其中单壁碳纳米管的分离 纯度并不高, 这意味着当DOC浓度低时, Tween 60 竞 争性过强导致分离单壁碳纳米管的选择性不高. 因此, 我们尝试了提高DOC的浓度, 以期提高分离的选择性.

当DOC浓度固定在 $0.04 \%$ 时, Tween 60 的浓度需 要达到DOC的 10 倍左右才能实现比较完全的分离(图 $3 \mathrm{~d}$ ), 高于DOC浓度在 $0.028 \%$ 时的 1.5 倍, 与预期相符, 但仍低于SDS-DOC复合表面活性剂体系. 提高DOC浓 

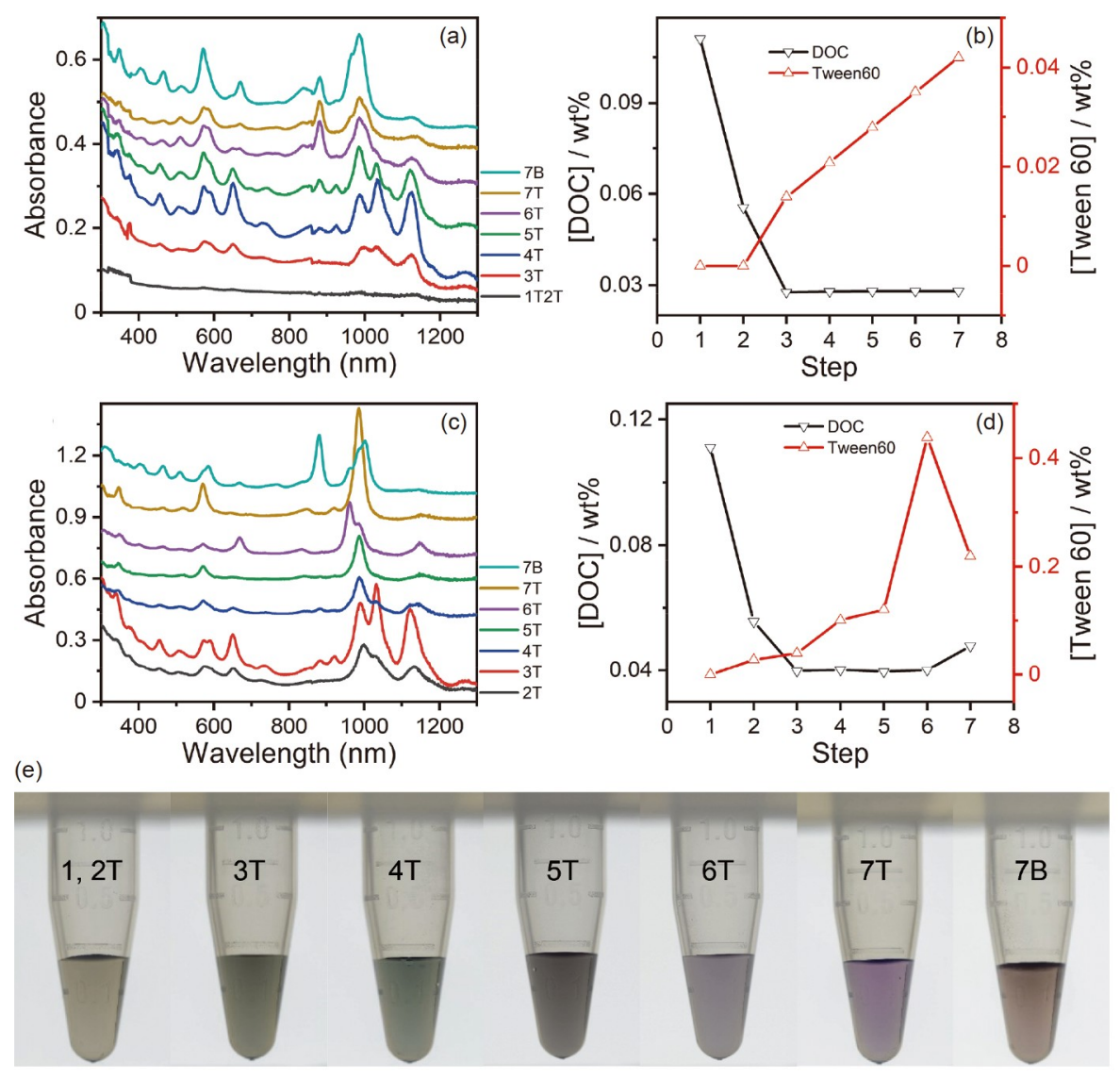

图 3 Tween 60-DOC复合表面活性剂体系对单壁碳纳米管的双水相分离结果. DOC浓度为 $0.028 \%$ 时双水相分离得到各相的 吸收光谱图(a) (由于1T与2T中基本无单壁碳纳米管, 故合并后测吸收光谱)及分离过程中表面活性剂浓度变化(b); DOC浓度为 $0.04 \%$ 时双水相分离得到各相的吸收光谱图(c) (1T中几乎无单壁碳纳米管)及分离过程中表面活性剂浓度变化(d); (e) Tween 60 -DOC $(0.028 \%)$ 体系双水相萃取过程的光学照片(网络版彩图)

Figure 3 ATP separation results using Tween 60-DOC. Absorption spectra of each top and bottom phases extracted (a) (since there were almost no SWCNTs in $1 \mathrm{~T}$ and $2 \mathrm{~T}$, they were mixed together for absorption measurement) and mass concentrations of surfactants of the whole ATP system containing Tween 60-DOC co-surfactant during the separation process (b) when DOC concentration was controlled at $\sim 0.028 \%$; absorption spectra of each top and bottom phases extracted (c) (there were almost no SWCNTs in 1T) and mass concentrations of surfactants of the whole ATP system containing Tween 60-DOC co-surfactant during the separation process (d) when DOC concentration was controlled at $\sim 0.04 \%$; (e) optical photographs of the ATP progress of Tween 60-DOC $(0.028 \%)$ system (color online).

度后，从吸收光谱中可以看到每一相中单壁碳纳米管 的手性种类更少，分离的选择性更高，尤其在7T中(图 $3 c)$, 得到了纯度极高的 $(6,5)$ 型碳纳米管. 因此，提高 $\mathrm{DOC}$ 的浓度可以提高分离的选择性，这也同时为提高 单壁碳纳米管的浓度以进行更大量的分离提供了方 便. 而SDS在水中的溶解度有限, 且其溶液极容易起 泡，不易提高浓度。若通过提高DOC浓度以期提高 SDS-DOC复合表面活性剂体系的分离效果，由于SDS 对DOC的竞争吸附较弱, 则实现分离所需的SDS浓度 也必定更高，预期将远高于DOC浓度为 $0.02 \%$ 时的 20 倍, 这在实际的双水相体系中是很难达到的.
我们发现，当DOC浓度为 $0.04 \%$ 时，在逐步提高 Tween 60浓度的过程中会发生界面聚集, 即有一部分 单壁碳纳米管聚集在上下两相的界面处, 不能完全从 下相转移分配至上相. 通过先稀释降低DOC浓度使单 壁碳纳米管全都分配到上相，再提高DOC的浓度将部 分单壁碳纳米管转移至下相或界面处可以解决该问 题, 图中7T就是通过这种方法得到的.

如图4所示, 比较Tween 60-DOC体系7T和SDSDOC体系10T的吸收光谱可见, 在400 600 $\mathrm{nm}$ 处的吸 收峰前者更低, 说明其中的金属性管含量更少，且在 $900 \mathrm{~nm}$ 附近含有更少的其他手性的单壁碳纳米管, 也 


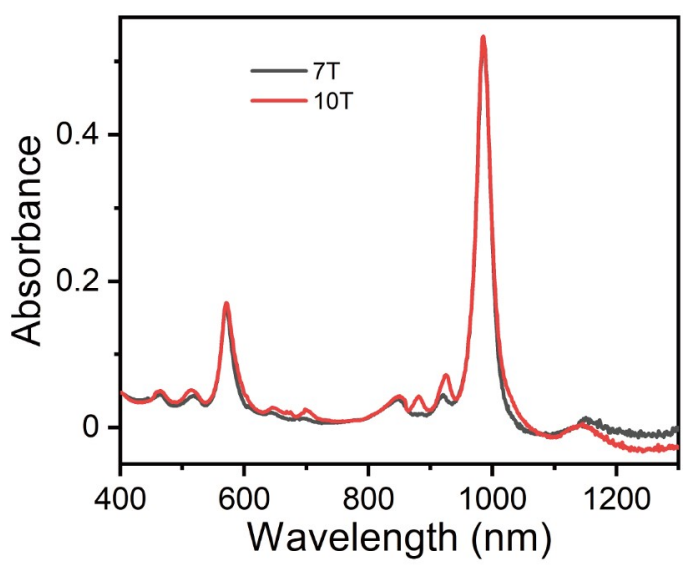

图 4 SDS-DOC体系10T与Tween 60-DOC $(0.04 \%)$ 体系7T $(6,5)$ 富集相吸收光谱的比较(网络版彩图)

Figure 4 The absorption spectra of $(6,5)$-enriched $10 \mathrm{~T}$ and $7 \mathrm{~T}$ obtained from SDS-DOC and Tween 60-DOC co-surfactant system, respectively (color online)

就是说Tween 60-DOC体系分出的第7个上相比SDSDOC 体系的第 10 个上相中的 $(6,5)$ 纯度更高，体现了 Tween 60在单一手性 $(6,5)$ 管分离中的优势.

\section{4 结论}

将非离子型表面活性剂Tween 60与DOC复配用于 双水相萃取分离单壁碳纳米管时，有明显的管径分离 趋势; 与SDS-DOC体系相比，在更少的分离步骤下就 可分离出纯度更高的(6,5)型碳纳米管，且不会像SDSDOC体系一样受到SDS 溶解度的限制，操作简便. Tween 60 起到与SDS相似的作用，即更倾向于包覆大 管径单壁碳纳米管且包覆后的单壁碳纳米管更疏水. 但Tween 60 与DOC对单壁碳纳米管的竞争包覆能力更 强，可在更高DOC浓度和更高碳纳米管浓度下进行分 离, 有利于实现碳纳米管的大量分离. 在双水相法中 使用Tween 60-DOC体系分离 $(6,5)$ 管，比SDS-DOC体

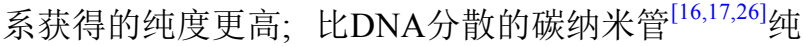
度略低，但成本要低得多; 该方法得到的 $(6,5)$ 管浓度 显著高于共轭高分子 ${ }^{[30]}$ 选择性抓取; 相比于凝胶色谱 法 $^{[14,31]}$ 分离, 此方法得到的(6,5)管不仅浓度更高, 也不 依赖于复杂的色谱装置. 综上，基于 Tween 60-DOC的 双水相分离更具实用性.

\section{参考文献}

1 Iijima S, Ichihashi T. Nature, 1993, 363: 603-605

2 Liu L, Han J, Xu L, Zhou J, Zhao C, Ding S, Shi H, Xiao M, Ding L, Ma Z, Jin C, Zhang Z, Peng LM. Science, 2020, 368: 850-856

3 Hills G, Lau C, Wright A, Fuller S, Bishop MD, Srimani T, Kanhaiya P, Ho R, Amer A, Stein Y, Murphy D, Arvind D, Chandrakasan A, Shulaker MM. Nature, 2019, 572: 595-602

4 Yomogida Y, Tanaka T, Zhang M, Yudasaka M, Wei X, Kataura H. Nat Commun, 2016, 7: 12056

5 Saha A, Gifford BJ, He X, Ao G, Zheng M, Kataura H, Htoon H, Kilina S, Tretiak S, Doorn SK. Nat Chem, 2018, 10: 1089-1095

8 Tu X, Manohar S, Jagota A, Zheng M. Nature, 2009, 460: 250-253

9 Arnold MS, Stupp SI, Hersam MC. Nano Lett, 2005, 5: 713-718

10 Arnold MS, Green AA, Hulvat JF, Stupp SI, Hersam MC. Nat Nanotech, 2006, 1: 60-65

11 Ghosh S, Bachilo SM, Weisman RB. Nat Nanotech, 2010, 5: 443-450

12 Moshammer K, Hennrich F, Kappes MM. Nano Res, 2009, 2: 599-606

13 Tanaka T, Jin H, Miyata Y, Fujii S, Suga H, Naitoh Y, Minari T, Miyadera T, Tsukagoshi K, Kataura H. Nano Lett, 2009, 9: 1497-1500

14 Liu H, Nishide D, Tanaka T, Kataura H. Nat Commun, 2011, 2: 309

15 Khripin CY, Fagan JA, Zheng M. J Am Chem Soc, 2013, 135: 6822-6825

16 Ao G, Streit JK, Fagan JA, Zheng M. J Am Chem Soc, 2016, 138: 16677-16685

17 Lyu M, Meany B, Yang J, Li Y, Zheng M. J Am Chem Soc, 2019, 141: 20177-20186

18 Fagan JA, Khripin CY, Silvera Batista CA, Simpson JR, Hároz EH, Hight Walker AR, Zheng M. Adv Mater, 2014, 26: 2800-2804

19 Li H, Gordeev G, Garrity O, Peyyety NA, Selvasundaram PB, Dehm S, Krupke R, Cambré S, Wenseleers W, Reich S, Zheng M, Fagan JA, Flavel BS. ACS Nano, 2020, 14: 948-963 
Fagan JA, Hároz EH, Ihly R, Gui H, Blackburn JL, Simpson JR, Lam S, Hight Walker AR, Doorn SK, Zheng M. ACS Nano, 2015, 9: 5377-5390

Gui H, Streit JK, Fagan JA, Hight Walker AR, Zhou C, Zheng M. Nano Lett, 2015, 15: 1642-1646

Wei L, Liu B, Wang X, Gui H, Yuan Y, Zhai S, Ng AK, Zhou C, Chen Y. Adv Electron Mater, 2015, 1: 1500151

Li H, Gordeev G, Garrity O, Reich S, Flavel BS. ACS Nano, 2019,13: 2567-2578

Zhang M, Khripin CY, Fagan JA, McPhie P, Ito Y, Zheng M. Anal Chem, 2014, 86: 3980-3984

Subbaiyan NK, Cambré S, Parra-Vasquez ANG, Hároz EH, Doorn SK, Duque JG. ACS Nano, 2014, 8: 1619-1628

Ao G, Khripin CY, Zheng M. J Am Chem Soc, 2014, 136: 10383-10392

Li Y, Lyu M. Sci Sin-Chim, 2020, 50: 1619-1636

Wenseleers W, Vlasov II, Goovaerts E, Obraztsova ED, Lobach AS, Bouwen A. Adv Funct Mater, 2004, 14: 1105-1112

Moore VC, Strano MS, Haroz EH, Hauge RH, Smalley RE, Schmidt J, Talmon Y. Nano Lett, 2003, 3: 1379-1382

Ozawa H, Ide N, Fujigaya T, Niidome Y, Nakashima N. Chem Lett, 2011, 40: 239-241

Liu H, Tanaka T, Urabe Y, Kataura H. Nano Lett, 2013, 13: 1996-2003

\title{
Aqueous two-phase extraction of single-walled carbon nanotubes using nonionic surfactant Tween 60
}

\author{
Shaochuang Chen ${ }^{1}$, Min Lyu ${ }^{1,2^{*}}$, Yan $\mathrm{Li}^{1 *}$ \\ ${ }^{1}$ Beijing National Laboratory for Molecular Science, Key Laboratory for the Physics and Chemistry of Nanodevices, State Key Laboratory of Rare \\ Earth Materials Chemistry and Applications, College of Chemistry and Molecular Engineering, Peking University, Beijing 100871, China \\ ${ }^{2}$ Peking University Shenzhen Institute, PKU-HKUST ShenZhen-HongKong Institution, Shenzhen 518057, China \\ *Corresponding authors (email: yanli@pku.edu.cn; lvmin@pku.edu.cn)
}

\begin{abstract}
Due to its excellent electronic and optical properties, single-walled carbon nanotubes (SWNTs) have attracted extensive attention. Since the property of SWNTs is determined by their structures, applications like highperformance devices require SWNTs with identical structures and uniform properties. Therefore, the sorting of SWNTs is of great significance. Aqueous two-phase (ATP) extraction is an emerging method that can be utilized to realize the structure sorting of SWNTs. ATP extraction is a repeatable and reliable technique that does not require state-of-the-art equipment. It is efficient and easy to scale up. In this article, by adjusting the concentration of sodium deoxycholate (DOC) and nonionic surfactant Tween 60, the diameter sorting of SWNTs and the separation of high purity $(6,5)$ tubes were achieved. The sorting can be carried out at a higher DOC concentration, which could improve the competitive coating between DOC and Tween 60 on the surface of SWNTs, and further improve the sorting efficiency of the cosurfactant system. The comparison of the separation results between the Tween 60-DOC surfactant system and the SDSDOC surfactant system shows that the introduction of nonionic surfactant Tween 60 is beneficial to the separation of higher purity $(6,5)$ tubes.
\end{abstract}

Keywords: single-walled carbon nanotubes, aqueous two-phase extraction, non-ionic surfactant

doi: $10.1360 /$ SSC-2021-0184 